OPEN ACCESS

Edited by:

Jie Hu,

The Ohio State University,

United States

Reviewed by:

Tony Kuo,

UCLA Fielding School of Public

Health, United States

Josef Mitáš

Palacký University, Czechia

*Correspondence: Noël C. Barengo nbarengo@fiu.edu

Specialty section:

This article was submitted to Life-Course Epidemiology and Social

Inequalities in Health,

a section of the journal

Frontiers in Public Health

Received: 29 January 2021

Accepted: 25 October 2021

Published: 24 November 2021

Citation:

Andrade L, Geffin R, Maguire M, Rodriguez P, Castro G, Alkhatib A and Barengo NC (2021) The Associations

Between Access to Recreational

Facilities and Adherence to the American Heart Association's Physical Activity Guidelines in US Adults.

Front. Public Health 9:660624.

doi: 10.3389/fpubh.2021.660624

\section{The Associations Between Access to Recreational Facilities and Adherence to the American Heart Association's Physical Activity Guidelines in US Adults}

\author{
Larissa Andrade ${ }^{1}$, Ryan Geffin ${ }^{1}$, Mark Maguire ${ }^{1}$, Pura Rodriguez ${ }^{1}$, Grettel Castro ${ }^{1}$, \\ Ahmad Alkhatib ${ }^{2}$ and Noël C. Barengo ${ }^{1,3,4 *}$
}

\begin{abstract}
'Department of Translational Medicine, Herbert Wertheim College of Medicine, Florida International University, Miami, FL, United States, ${ }^{2}$ School of Health and Life Sciences, Teesside University, Middlesbrough, United Kingdom, ${ }^{3}$ Department of Public Health, Faculty of Medicine, University of Helsinki, Helsinki, Finland, ${ }^{4}$ Department of Health Policy and Management, Robert Stempel College of Public Health and Social Work, Florida International University, Miami, FL, United States
\end{abstract}

Physical activity decreases the risk of long-term health consequences including cardiac diseases. According to the American Health Association (AHA), adults should perform at least 75 min of vigorous physical activity (PA) or 150 min of moderate PA per week to impact long-term health. Results of previous studies are varied and have yet to integrate perceived access to facilities with AHA PA guidelines. We investigated whether access to free or low-cost recreational facilities was associated with meeting the AHA PA guidelines.

Methodology: This cross-sectional study utilized data extracted from the Family Life, Activity, Sun, Health, and Eating (FLASHE) database collected in $2017(n=1,750)$. The main exposure variable was access to free or low-cost recreational facilities. The main outcome variable was meeting the AHA guidelines of 150 min moderate PA or 75 min vigorous PA per week. Covariates included age, sex, level of education, overall health, BMI, ethnicity, hours of work per week, income, and time living at current address. Unadjusted and adjusted logistic regression analysis were used to calculate measures of odds ratio (OR) and corresponding 95\% confidence interval (Cl).

Results: Of the 1,750 included participants, $61.7 \%(n=1,079)$ reported to have access to recreational facilities. Of those with access to facilities, 69.9\% met AHA PA guidelines while $30.4 \%$ did not. After adjusting for covariates, participants who reported access to recreational facilities were $42 \%$ more likely to meet AHA PA guidelines compared with participants who did not (adjusted OR 1.42; 95\% Cl 1.14-1.76). Secondary results suggest that healthier individuals were more likely to have met AHA PA guidelines.

Conclusions: Having access to free or low-cost recreational facilities such as parks, walking trails, bike paths and courts was associated with meeting the AHA PA guidelines. 
Increasing prevalence and awareness of neighborhood recreational facilities could assist in access to these facilities and increase the ability of individuals to meet AHA PA guidelines. Future research should determine which types of recreational facilities impact physical activity strongest and discover methods of increasing their awareness.

Keywords: open space access, recreational facilities, built environment, sedentarism, compliance

\section{INTRODUCTION}

Physical activity (PA) is known to decrease the risk of developing long term chronic diseases such as cardiovascular disease. Inadequate PA has long been associated with disease-related mortality and increased healthcare costs. Recent estimates in the US reported about $10 \%$ of pre-mature deaths were associated with inadequate $\mathrm{PA}$, and $\$ 117$ billion in annual healthcare costs (1). The current recommendation of the American Heart Association (AHA) for adults is to perform at least $150 \mathrm{~min}$ of moderate PA or $75 \mathrm{~min}$ of vigorous PA per week to have an impact on long term health (2). However, the U.S. Department of Health and Human Services (HHS) estimated that more than $80 \%$ of adults do not currently meet such guidelines (3). In such, it is essential to understand the factors associated with meeting current US guidelines among US populations.

One of the commonly accepted factors for increasing PA participation among populations is having safe and accessible recreational parks around their community (4). While it is known that expansive park networks in neighborhood-built environments are positively associated with multiple aspects of health, well-being, and quality of life (4), populations' access and PA participation in such neighborhood parks is influenced by different variables such as individuals' ability, motivation to be physically active, and parks and leisure centers' proximity to their homes (5). For example, only adolescents living in neighborhoods with available recreational facilities have reported increased frequency and duration of performing PA (6). Insufficient numbers of parks and leisure centers have also been reported for US homes, with only one of five US homes having parks and leisure centers within half a mile radius (3). Nonetheless, reported association between increased PA levels and facility numbers within a neighborhood is inconclusive (7-11), showing either statistically positive (12), negative (7), or no significant association between the two variables $(8$, 9, 13). For example, Stewart et al., found no association between park's proximity and PA levels among an urban, mostly white, well-educated sample population that was well-served by neighborhood parks (9), though neither their socio-economic status nor their PA access was assessed. West et al., only focused on the largest American cities whilst reporting a positive correlation between park density and PA levels, which may not therefore be generalizable to smaller cities or rural areas (10).

\footnotetext{
Abbreviations: AHA, American Heart Association; BMI, Body Mass Index; CI, Confidence Interval; FLASHE, Family Life, Activity, Sun, Health, and Eating; HHS, U.S. Department of Health and Human Services; OR, Odds Ratio; PA, Physical Activity; Ref, Reference Group; Rs, Spearman's Rank Correlation Coefficient; US, United States; WHO, World Health Organization.
}

Whether and how PA participation is determined by facility access across US populations in large cities are largely unknown.

The objective of this study was to investigate whether access to free or low-cost recreational facilities was associated with meeting the AHA PA guidelines among US adults.

\section{MATERIALS AND METHODS \\ Study Design and Population}

This was an analytical cross-sectional study utilizing demographics, built environment characteristics, and PA level data obtained from the Family Life, Activity, Sun, Health, and Eating (FLASHE) study conducted by the National Cancer Institute. The survey results in this publicly available online database encompassed a population of adult $(N=1,839)$ in the United States in 2017. A non-probability sample was recruited from all U.S. regions through the Ipsos Consumer Opinion Panel, a national market research firm. Based on sex, census division, household income and size, and race/ethnicity, eligible participants were balanced in the U.S. population. Within each household, one adolescent and one parent were randomly selected from eligible household members. Parents were considered eligible if there were at least 18 years of age and lived with at least one child aged between 12 and 17 years of age for $>50 \%$ of the time. However, in this study we only included the parents. Via three surveys, these individuals were asked questions on demographics, health status, PA status, diet and built environment regarding both themselves and their adolescent (14). The main inclusion criteria included age of 18 years or above, participation in the 2017 FLASHE survey; and response to survey questions being studied including "My neighborhood has several FREE or LOW-COST recreation facilities, such as parks, walking trails, bike paths, recreation centers, playgrounds, etc.", and one of the following: "How much time did you usually spend doing vigorous PA on one of those days?", "How much time did you usually spend doing MODERATE PA on one of those days?" (15). Similarly, participants were excluded from the study if they either missed responses to the survey question assessing the independent variable described below, or if they did not have a response to any one of the two questions addressing the dependent variable.

\section{Variables}

The main independent variable in this study was accessibility to free or low-cost recreational facilities. Perceived access to free or low-cost recreational facilities was assessed by the question "My neighborhood has several FREE or LOW-COST recreation facilities, such as parks, walking trails, bike paths, recreation centers, playgrounds, etc." Participants could either respond 
"strongly agree," "somewhat agree," "somewhat disagree," or "strongly disagree." To create a dichotomous variable, the answer choices "strongly agree" and "somewhat agree" were combined into "agree"; and "strongly disagree" and "somewhat disagree" into "disagree."

The main outcome variable was adherence to the AHA national PA guidelines. These guidelines recommend that adults should participate in more than 150 min of moderate intensity PA or more than $75 \mathrm{~min}$ of vigorous intensity PA per week (2). The self-reported quantitative weekly time spent doing walking, moderate or vigorous PA into one of two categorical variables: (1) at or above national guidelines or (2) below national guidelines. Quantitative data on vigorous activity were collected by the following questions: "During the LAST 7 DAYS, on how many days did you do VIGOROUS PA like heavy lifting, digging, aerobics, or fast bicycling?" and "How much time did you usually spend doing vigorous PA on one of those days?". The amount of moderate PA was assessed by: "During the LAST 7 DAYS, on how many days did you do MODERATE PA like carrying light loads, bicycling at a regular pace, or doubles tennis? Do not include walking," and "How much time did you usually spend doing MODERATE PA on one of those days?", respectively. Individuals were considered above or equal to guidelines if they meet the guideline criteria for either vigorous or moderate PA. Those who do not meet guidelines for either of these two categories were categorized as below national PA guidelines.

This study included the covariates age, sex, level of education, overall health, BMI, ethnicity, employment status, hours of work per week, income, and number of years living at current residence. Age was assessed in the survey using the following categories: $18-34,35-44,45-59$, and $60+$ years-of-age (16). Sex was categorized into male or female. Levels of education were re-categorized into (i) high school or less (ii), some college but not a college degree, (iii) or a 4-year college degree or higher. Overall health status was re-categorized into excellent, very good, good, or fair/poor. BMI was calculated with height and weight and was categorized according to WHO criteria (17). A BMI $<18.5 \mathrm{~kg} / \mathrm{m}^{2}$ was considered underweight. A BMI between 18.5 and $24.99 \mathrm{~kg} / \mathrm{m}^{2}$ was considered normal weight. A BMI between 25 and $29.99 \mathrm{~kg} / \mathrm{m}^{2}$ was considered overweight. A BMI $30 \mathrm{~kg} / \mathrm{m}^{2}$ and above was considered obese. Race/ethnicity was divided into four groups: Hispanic, Non-Hispanic Black, NonHispanic White, and others. Work status was categorized into not working, $0-30 \mathrm{~h}, 31-40 \mathrm{~h}$, or $41+\mathrm{h}$. Income was dichotomized into $\geq \$ 100,000$ and $<\$ 100,000$.

\section{Statistical Analysis}

The data was analyzed utilizing Stata 16 software package (StatCorp LLC, College Station, Texas) (18). Primarily, a descriptive analysis was implemented to check the distribution of each variable. A chi-squared was used to perform a bivariate analysis of categorical data according to the main independent and the outcome variable to check for possible confounders. A collinearity check was then performed to check whether there is too much correlation between the variables. Lastly, unadjusted and adjusted logistic regression analyses were used to calculate the odds ratios and corresponding $95 \%$ confidence intervals. The
Hosmer-Lemeshow test was used to assess the goodness-of-fit of the logistic regression models.

\section{RESULTS}

Table 1 presents the characteristics of the study participants of the 2017 FLASHE survey according to access to free or lowcost recreational facilities $(n=1,750)$. There was no statistically significant difference in the distribution of the age groups according to whether they had access to free or low-costs facilities or whether they did not (chi-square $p$-value 0.119). However, there was a statistically significantly higher proportion of men in those with access to free or low-cost facilities (30.6\%) compared with those without access $(19.4 \%$; $p$-value $<0.001)$. In addition, participants' race/ethnicity, health status, BMI, education, work status and household income were statistically significantly different among those with access to free or low-cost recreational facilities compared with those without access ( $p$-values $<0.05$ ). For instance, those with access to recreational facilities had a higher proportion of being normal weight (39.3\%) than those without access (31.5\%). Moreover, those with access tend to have a higher perceived excellent or very good health compared with participants without access to free or low-cost recreational facilities ( 59.5 vs. $52.7 \%$ ). Finally, $24 \%$ of the participants who had access to recreational facilities had a household income of at least 100,000 USD/year whereas the corresponding proportion was $15.1 \%$ in those without access ( $p$-value $<0.001$ ).

The unadjusted and adjusted associations between respondent characteristics and meeting AHA PA guidelines, which were stratified by access to free or low-cost recreational facilities are presented in Table 2. Before adjusting for covariates, participants who had access to free or low-cost recreational facilities had an odds ratio (OR) of meeting AHA PA guidelines of 1.63 (95\% CI 1.33-1.99) when compared with participants who did not. After adjustment for the covariates, participants who had access to free or low-cost recreational facilities had statistically significantly higher odds of meeting the AHA PA guidelines compared with participants who disagreed with this statement [adjusted OR (aOR) 1.42; 95\% CI 1.14-1.76]. The adjusted odds of meeting the AHA PA guidelines for the 35-44 age group was 0.77 (95\% CI $0.54-1.11$ ), for the age $45-59$ age group 1.01 (95\% CI $0.57-1.21)$ and for those $\geq 60$ years-old 0.88 (95\% CI $0.31-1.27$ ) when compared with participants age $18-34$ years-old. Participants who reported being male were 1.99 times more likely to meet the AHA PA guidelines compared with females (aOR 1.99; 95\% CI 1.49-2.65). Being non-Hispanic white, Hispanic (aOR 1.31; 95\% CI 0.83-2.07), non-Hispanic black (aOR 0.85; 95\% CI $0.64-1.14$ ) or other (aOR $0.8 ; 5$ 95\% CI $0.54-1.35$ ) was not associated with meeting AHA PA guidelines. Regarding health status, participants who reported excellent health status were 2.42 times more likely to meet the AHA PA guidelines (aOR 2.42; 95\% CI 1.66-3.52), while those who reported very good health status were 1.47 times more likely to meet them (aOR 1.47; 95\% CI 1.14-1.90) when compared with those with good health status. Furthermore, obese participants compared with those having a normal BMI had 39\% reduced odds to 
TABLE 1 | Characteristics distribution of the 2017 FLASHE participants with and without access to free or low-cost recreational facilities.

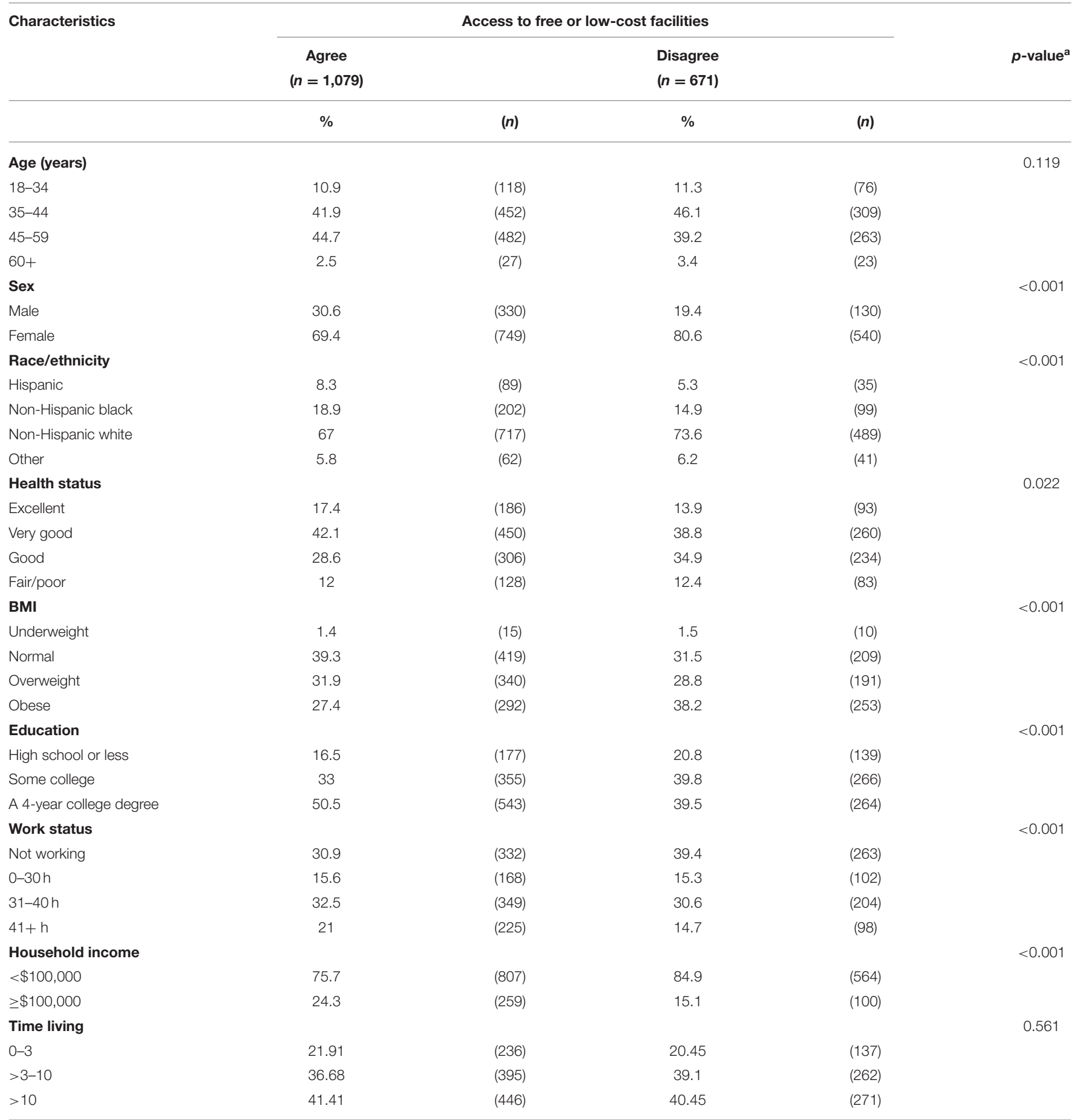

${ }^{a}$ Chi-square test p-value.

meet PA guidelines (aOR: 0.61; 95\% CI 0.46-0.80). Regarding education, having an educational level of high school or less, some college (aOR 1.09; 95\% CI 0.81-1.47), or 4-year college degree (aOR 1.28; 95\% CI 0.94-1.75) were all not associated with meeting the AHA PA guidelines. Household income status was not associated with increased or decreased likelihood of meeting the guidelines (aOR 1.32; 95\% CI 0.98-1.79).

\section{DISCUSSION}

The main finding of the present study is that access to free or low-cost recreational facilities was positively associated with meeting the AHA PA guidelines in US adults. However, such association is also affected by sex, BMI, and health status, which were all associated with complying with the AHA PA 
TABLE 2 | Unadjusted and adjusted association between access to free or low-cost recreational facilities and meeting the American Heart Association's Physical Activity Guidelines for Americans, 2017 FLASHE survey.

\begin{tabular}{|c|c|c|}
\hline \multirow[t]{3}{*}{ Characteristics } & \multicolumn{2}{|c|}{$\begin{array}{l}\text { Meeting American Heart Association Phys } \\
\text { Activity Guidelines }\end{array}$} \\
\hline & Unadjusted & Adjusted \\
\hline & $\mathrm{OR}^{\mathrm{a}}\left(95 \% \mathrm{Cl}^{\mathrm{b}}\right)$ & $\operatorname{aOR}^{a}\left(95 \% \mathrm{Cl}^{b}\right)$ \\
\hline \multicolumn{3}{|c|}{ Access to free or low-cost facilities } \\
\hline Disagree & $\operatorname{Ref}^{\mathrm{C}}$ & $\operatorname{Ref}^{\mathrm{C}}$ \\
\hline Agree & $1.63(1.33-1.99)$ & $1.42(1.14-1.76)$ \\
\hline \multicolumn{3}{|l|}{ Sex } \\
\hline Female & $\operatorname{Ref}^{\mathrm{C}}$ & $\operatorname{Ref}^{\mathrm{C}}$ \\
\hline Male & $2.23(1.74-2.85)$ & $1.99(1.49-2.65)$ \\
\hline \multicolumn{3}{|l|}{ Health status } \\
\hline Good & $\operatorname{Ref}^{\mathrm{C}}$ & $\operatorname{Ref}^{\mathrm{C}}$ \\
\hline Excellent & $2.89(1.05-4.06)$ & $2.42(1.66-3.52)$ \\
\hline Very good & $1.68(1.33-2.12)$ & $1.47(1.14-1.90)$ \\
\hline Fair/poor & $0.60(0.44-0.83)$ & $0.70(0.50-0.99)$ \\
\hline \multicolumn{3}{|l|}{ BMI } \\
\hline Normal & $\operatorname{Ref}^{\mathrm{C}}$ & $\operatorname{Ref}^{\mathrm{C}}$ \\
\hline Underweight & $0.63(0.27-1.45)$ & $0.59(0.23-1.48)$ \\
\hline Overweight & $0.74(0.58-0.96)$ & $0.82(0.62-1.08)$ \\
\hline Obese & $0.41(0.32-0.53)$ & $0.61(0.46-0.80)$ \\
\hline
\end{tabular}

${ }^{a}$ Age, Sex, Level of education, Overall health, BMI, Ethnicity, Hours of work per week, Household Income, Time living at current address.

${ }^{b}$ Odds ratio.

${ }^{c}$ Confidence interval.

${ }^{d}$ Reference group; p-value of the Hosmer-Lemeshow test for the adjusted model > 0.05.

guidelines. When controlling for covariates, males were twice likely to meet AHA PA guidelines compared with females, and participants with obesity were $39 \%$ less likely to meet AHA PA guidelines compared with those with normal BMI; while those with excellent health status were 2.42 times more likely to meet AHA PA guidelines when compared with those who reported a good health.

Our findings on subjective availability of outdoor recreational facilities and higher levels of total leisure-time PA are in line with previously reported associations. For example, in a survey in five European urban regions, Mackenbach et al. found a $25 \%$ difference in weekly min of total leisure-time PA between individuals with and without availability of outdoor recreational facilities. They postulated that the availability of these facilities seemed to be an important underlying mechanism for increased populations' $\mathrm{PA}$, and the proximity was the main motivator for using recreational facilities (19). Several other reports have also showed that individuals who resided in neighborhoods with higher number of public parks and recreational facilities were more likely to be regularly physically active (7-11). One study described an increased amount of park-based PA in people with two parks within one mile (aOR 2.29) and three or more parks in one mile (OR 2.53) compared with those without any parks within one mile radius (8). West et al. reported that significant, positive correlations between park density and reported PA
( $r=0.37, p<0.01)$, and between park density and reported performing regular exercise $(r=0.35, p<0.01)$ (10). While availability of public parks is now an established determinant in meeting PA guidelines, the scientific literature is inconsistent when analyzing an association between the proximity of public parks and PA levels. Kaczynski et al. reported that distance was not significantly associated with an increased likelihood of PA at the park $(8,13)$. Conversely, Cohen et al. reported that people living within one mile of the park averaged $38 \%$ more regular exercise sessions per week compared to those living further (IRR $1.38 ; 95 \%$ CI $1.04-1.84$ ) and were four times as likely to visit the park once a week or more than those living further away (12). Therefore, reporting on the access to free and low-cost PA facilities and its positive association with meeting PA AHA guidelines in our study provides a new approach for increasing PA and associated long term health benefits in US populations.

Our study relied on a more subjective reporting when observing the association between access to free and lowcost recreational facilities and an increased likelihood of being physically active among US adults. An important distinction between the subjective (e.g., availability dependent on factors such as cost, safety, and hygiene) and objective availability (e.g., actual access or use) of recreational facilities has been previously reported with a significant association between the subjective availability of recreational facilities and leisure-time PA, however when the analysis was based on the objective availability (or reported use) of recreational facilities the association was not statistically significant (19). This may be due in part because individuals who report access to outdoor recreational facilities do so because they use them regularly-resulting in stronger associations with the perceived measure of PA levels (20).

The secondary outcomes of our analysis suggest that the healthier a person is the more likely they are to have met AHA PA guidelines $(\mathrm{aOR}=2.42$ and 1.47 for Excellent and Good Health, respectively), which was irrespective of the participants' age (Table 1). This may be explained by increased time spent exercising which has previously been associated with better health status (21). Therefore, increased time spent and awareness among those with perceived poor health is necessary when promoting PA access across US populations. Looking at BMI specifically, individuals with obesity were significantly less likely to meet the national PA guidelines which is contradictory to Lee et al.'s finding of obese individuals (BMI 25 and above) having no difference in level of PA when compared with individuals who are not obese (22). Our results most likely differ due to a difference in definition of obesity (over 25 BMI Asian cutoff point vs. over $30 \mathrm{BMI}$ in US) but can also be explained by various reasons including lack of social support, lack of time, choosing a sedentary lifestyle, or lack of motivation to use PA facilities $(23,24)$. Studies that integrated similar BMI stratification such as Hemmingsson et al. found similar results to ours of a clear association between BMI and level of PA (25). Sex had the largest impact on the odds of meeting PA guidelines where men were twice at odds of meeting PA guidelines than women. This finding is in line with previous studies that describe increased PA in males compared to females (26). It has been proposed in past studies that societal influences are stronger on males than 
females regarding PA which can explain the increased likelihood of meeting guidelines for men (27).

Naturally, our study has some limitations. First, our crosssectional study design lacks the ability to implement causality. While we can illustrate an association between two variables, we are unable to correlate the two due in part to the possibility of reverse causality. The study design also introduces the possibility of various types of bias. Our study measured PA by a selfreported questionnaire, which may over- or underestimate true PA behavior. Thus, there is potential for social acceptability biases. This may be illustrated in part by the larger than expected percentage of individuals that met requirements and the few impossible and few possible but improbable outcomes reported. Nevertheless, the PA questions in this study were validated against an objective PA accelerometer data $(r=0.52, p<0.01)$ (28). Similarly, while this study makes an important distinction from past studies in that it reports people's access to free or low-cost facilities, we recognize that it is somewhat vague in its definition of accessibility. While it is important to recognize that there is an association between access and PA, there is no concrete quantitative data to be used by local governments to best implement new policies. A strength of this study was that it included a national sample from a large, regionally diverse sample of the U.S. population, although not nationally representative. The national sample allowed taking into consideration variation in environmental contexts, socio-demographics, and behaviors. For example, access to recreational facilities vary between most U.S. states. Therefore, using a national sample allows for more generalizable results than a study located in a specific city or state.

In conclusion, our data provides various recommendations that can be extrapolated for use by health professionals, public health officials and the public. We recommend increasing the

\section{REFERENCES}

1. Piercy KL, Troiano RP, Ballard RM, Carlson SA, Fulton JE, Galuska DA, et al. The physical activity guidelines for Americans. JAMA. (2018) 320:20208. doi: 10.1001/jama.2018.14854

2. American Heart Association Recommendations for Physical Activity in Adults and Kids. Available online at: https://www.heart.org/en/healthy-living/fitness/ fitness-basics/aha-recs-for-physical-activity-in-adults (accessed October 31, 2021).

3. Secretary, HHS Office of the, and Fitness \& Nutrition President's Council on Sports. Facts \& Statistics. HHS.gov, US Department of Health and Human Services, (2017). Available online at: www.hhs.gov/fitness/resource-center/facts-and-statistics/index.html (accessed October 31, 2021).

4. Larson LR, Jennings V, Cloutier SA. Public parks and wellbeing in urban areas of the United States. PLoS ONE. (2016) 11:e0153211. doi: 10.1371/journal.pone.0153211

5. Wiseman KP, Patel M, Dwyer LA, Nebeling LC. Perceived weight and barriers to physical activity in parent-adolescent dyads. Health Psychol. (2018) 37:76774. doi: 10.1037/hea0000635

6. Gavand KA, Cain KL, Conway TL, Saelens BE, Frank LD, Kerr J, et al. Associations between neighborhood recreation environments and adolescent physical activity. J Phys Act Health. (2019) 16:880-5. doi: 10.1123/jpah.2018-0556

7. Gidlow C, Cerin E, Sugiyama T, Adams MA, Mitas J, Akram M, et al. Objectively measured access to recreational destinations and leisure-time availability of facilities such as parks, trails, fields, and courts in various neighborhoods, and to increase awareness of the availability of these facilities, perhaps via targeted social media, newspapers, magazines, and local television networks so that PA is promoted across wider populations especially females and those with poorer health conditions including those with obesity. Future studies could focus on improving access to free or low-cost recreational PA facilities by understanding individuals' barriers and facilitators to using such facilities and using more objective PA assessment approaches.

\section{DATA AVAILABILITY STATEMENT}

Publicly available datasets were analyzed in this study. This data can be found here: https://cancercontrol.cancer.gov/brp/hbrb/ flashe-study.

\section{AUTHOR CONTRIBUTIONS}

LA, RG, MM, AA, and NB contributed to the conception and design of the study. GC and PR supervised data collection. LA, $\mathrm{RG}$, and MM organized the dataset and wrote the first draft of the manuscript. LA, RG, MM, GC, and PR performed the statistical analysis. AA, GC, PR, and NB revised the manuscript. All authors contributed to manuscript revision, read and approved the submitted version.

\section{ACKNOWLEDGMENTS}

We would like to thank all study participants of the Family Life, Activity, Sun, Health, and Eating (FLASHE) database, the National Cancer Institute, and Florida International University.

physical activity: associations and demographic moderators in a six-country study. Health Place. (2019) 59:102196. doi: 10.1016/j.healthplace.2019.102196

8. Kaczynski AT, Besenyi GM, Stanis SA, Koohsari MJ, Oestman KB, Bergstrom $\mathrm{R}$, Potwarka LR, et al. Are park proximity and park features related to park use and park-based physical activity among adults? Variations by multiple socio-demographic characteristics. Int J Behav Nutr Phys Act. (2014) 11:146. doi: 10.1186/s12966-014-0146-4

9. Stewart OT, Moudon AV, Littman AJ, Seto E, Saelens BE. Why neighborhood park proximity is not associated with total physical activity. Health Place. (2018) 52:163-9. doi: 10.1016/j.healthplace.2018.05.011

10. West ST, Shores KA, Mudd LM. Association of available parkland, physical activity, and overweight in America's largest cities. J Public Health Manag Pract. (2012) 18:423-30. doi: 10.1097/PHH.0b013e3182 $38 \mathrm{ea} 27$

11. Adams MA, Frank LD, Schipperijn J, Smith G, Chapman J, Christiansen $\mathrm{LB}$, et al. International variation in neighborhood walkability, transit, and recreation environments using geographic information systems: the IPEN adult study. Int J Health Geogr. (2014) 13:43. doi: 10.1186/1476-072X-13-43

12. Cohen DA, McKenzie TL, Sehgal A, Williamson S, Golinelli D, Lurie N. Contribution of public parks to physical activity. Am J Public Health. (2007) 97:509-14. doi: 10.2105/AJPH.2005.072447

13. Kaczynski AT, Potwarka LR, Saelens BE. Association of park size, distance, and features with physical activity in neighborhood parks. Am J Public Health. (2008) 98:1451-6. doi: 10.2105/AJPH.2007.129064

14. Nebeling LC, Hennessy E, Oh AY, Dwyer LA, Patrick H, Blanck HM, et al. The FLASHE study: survey development, dyadic 
perspectives, and participant characteristics. Am J Prev Med. (2017) 52:839-48. doi: 10.1016/j.amepre.2017.01.028

15. Oh AY, Davis T, Dwyer LA, Hennessy E, Li T, Yaroch AL, et al. Recruitment, enrollment, and response of parent-adolescent dyads in the FLASHE study. Am J Prev Med. (2017) 52:849-55. doi: 10.1016/j.amepre.2016.11.028

16. Division of Cancer Control PS, National Cancer Institute. FLASHE Data Terms of Use. Available online at: https://cancercontrol.cancer.gov/sites/ default/files/2020-06/parent_demo_puf_instrument.pdf (accessed July 07, 2021).

17. Body Mass Index - BMI. Available online at: https://www.euro.who.int/en/ health-topics/disease-prevention/nutrition/a-healthy-lifestyle/body-massindex-bmi/ (accessed October 31, 2021).

18. StataCorp. Stata Statistical Software: Release 16. College Station, TX: StataCorp LLC (2019).

19. Mackenbach JD, Matias de Pinho MG, Faber E, Braver ND, de Groot $\mathrm{R}$, Charreire $\mathrm{H}$, et al. Exploring the cross-sectional association between outdoor recreational facilities and leisure-time physical activity: the role of usage and residential self-selection. Int J Behav Nutr Phys Activ. (2018) 15:55. doi: 10.1186/s12966-018-0689-x

20. Cerin E, Vandelanotte C, Leslie E, Merom D. Recreational facilities and leisure-time physical activity: an analysis of moderators and self-efficacy as a mediator. Health Psychol. (2008) 27:S12635. doi: 10.1037/0278-6133.27.2(Suppl.).S126

21. Granger E, Williams G, Di Nardo F, Harrison A, Verma A. The relationship between physical activity and self-rated health status in European adolescents: results of the EURO-URHIS 2 survey. Eur J Public Health. (2017) 27(Suppl. 2):107-11. doi: 10.1093/eurpub/ckw177

22. Lee SA, Ju YJ, Lee JE, Hyun IS, Nam JY, Han KT, et al. The relationship between sports facility accessibility and physical activity among Korean adults. BMC Public Health. (2016) 16:893. doi: 10.1186/s12889-016-3574-Z

23. Ashton LM, Hutchesson MJ, Rollo ME, Morgan PJ, Collins CE. Motivators and barriers to engaging in healthy eating and physical activity. Am J Mens Health. (2017) 11:330-43. doi: 10.1177/1557988316680936

24. Andajani-Sutjahjo S, Ball K, Warren N, Inglis V, Crawford D. Perceived personal, social and environmental barriers to weight maintenance among young women: a community survey. Int J Behav Nutr Phys Act. (2004) 1:15. doi: 10.1186/1479-5868-1-15

25. Hemmingsson E, Ekelund U. Is the association between physical activity and body mass index obesity dependent? Int J Obes (Lond). (2007) 31:6638. doi: $10.1038 /$ sj.ijo. 0803458

26. Craft BB, Carroll HA, Lustyk MK. Gender differences in exercise habits and quality of life reports: assessing the moderating effects of reasons for exercise. Int J Lib Arts Soc Sci. (2014) 2:65-76.

27. Telford RM, Telford RD, Olive LS, Cochrane T, Davey R. Why are girls less physically active than boys? Findings from the LOOK longitudinal study. PLoS ONE. (2016) 11:e0150041. doi: 10.1371/journal.pone.01 50041

28. Saint-Maurice PF, Kim Y, Hibbing P, Oh AY, Perna FM, Welk GJ Calibration and validation of the youth activity profile: the FLASHE study. Am J Prev Med. (2017) 52:880-7. doi: 10.1016/j.amepre.2016. 12.010

Conflict of Interest: The authors declare that the research was conducted in the absence of any commercial or financial relationships that could be construed as a potential conflict of interest.

Publisher's Note: All claims expressed in this article are solely those of the authors and do not necessarily represent those of their affiliated organizations, or those of the publisher, the editors and the reviewers. Any product that may be evaluated in this article, or claim that may be made by its manufacturer, is not guaranteed or endorsed by the publisher.

Copyright (c) 2021 Andrade, Geffin, Maguire, Rodriguez, Castro, Alkhatib and Barengo. This is an open-access article distributed under the terms of the Creative Commons Attribution License (CC BY). The use, distribution or reproduction in other forums is permitted, provided the original author(s) and the copyright owner(s) are credited and that the original publication in this journal is cited, in accordance with accepted academic practice. No use, distribution or reproduction is permitted which does not comply with these terms. 